\title{
Rapid discrimination of Enterococcus faecium strains using phenotypic analytical techniques
}

DOI:

10.1039/C6AY02326F

Document Version

Accepted author manuscript

Link to publication record in Manchester Research Explorer

\section{Citation for published version (APA):}

AlMasoud, N., Xu, Y., Ellis, D., Rooney, P., Turton, J., \& Goodacre, R. (2016). Rapid discrimination of Enterococcus faecium strains using phenotypic analytical techniques. Analytical Methods, 8(42), 7603-7613. https://doi.org/10.1039/C6AY02326F

\section{Published in:}

Analytical Methods

\section{Citing this paper}

Please note that where the full-text provided on Manchester Research Explorer is the Author Accepted Manuscript or Proof version this may differ from the final Published version. If citing, it is advised that you check and use the publisher's definitive version.

\section{General rights}

Copyright and moral rights for the publications made accessible in the Research Explorer are retained by the authors and/or other copyright owners and it is a condition of accessing publications that users recognise and abide by the legal requirements associated with these rights.

\section{Takedown policy}

If you believe that this document breaches copyright please refer to the University of Manchester's Takedown Procedures [http://man.ac.uk/04Y6Bo] or contact uml.scholarlycommunications@manchester.ac.uk providing relevant details, so we can investigate your claim.

\section{OPEN ACCESS}


1 Rapid discrimination of Enterococcus faecium strains using phenotypic

2 analytical techniques and advanced chemometrics

3

$4 \quad$ Najla AlMasoud, ${ }^{\mathrm{a}}$ Yun Xu, ${ }^{\mathrm{a}}$ David I. Ellis, ${ }^{\mathrm{a}}$ Paul Rooney, ${ }^{\mathrm{b}}$ Jane F. Turton, ${ }^{\mathrm{c}}$ Royston

5 Goodacre ${ }^{\text {a \# }}$

6

$7{ }^{a}$ School of Chemistry and Manchester Institute of Biotechnology, University of

8 Manchester, 131 Princess Street, Manchester, M1 7DN, UK.

$9 \quad{ }^{b}$ Belfast City Hospital, Belfast, 51 Lisburn Rd, BT9 7AB, UK.

$10{ }^{c}$ Antimicrobial Resistance and Healthcare Associated Infection Reference Unit,

11 National Infection Service, Public Health England, London, NW9 5EQ, UK.

$12 \quad$ "Correspondence to Roy Goodacre: roy.goodacre@manchester.ac.uk

13

14 Keywords:

15 Enterococcus faecium, classification, MALDI-TOF-MS, FT-IR, Raman, pulsed-field 16 gel electrophoresis, chemometrics. 


\section{ABSTRACT}

18 Clinical isolates of glycopeptide resistant enterococci (GRE) were used to compare

19 three rapid phenotyping and analytical techniques. Fourier transform infrared (FT-

20 IR) spectroscopy, Raman spectroscopy and matrix-assisted laser

21 desorption/ionization time-of-flight mass spectrometry (MALDI-TOF-MS) were

22 used to classify 35 isolates of Enterococcus faecium representing 12 distinct pulsed-

23 field gel electrophoresis (PFGE) types. The results show that the three analytical

24 techniques provide clear discrimination among enterococci at both the strain and

25 isolate levels. FT-IR and Raman spectroscopic data produced very similar bacterial

26 discrimination, reflected in the Procrustes distance between the datasets $(0.2125$ -

27 0.2411, $p<0.001$ ); however, FT-IR data provided superior prediction accuracy to

28 Raman data with correct classification rates (CCR) of $89 \%$ and $69 \%$ at the strain

29 level, respectively. MALDI-TOF-MS produced slightly different classification of

30 these enterococci strains also with high CCR (78\%). Classification data from the

31 three analytical techniques were consistent with PFGE data especially in the case of

32 isolates identified as unique by PFGE. This study presents phenotypic techniques as

33 a complementary approach to current methods with a potential for high-throughput

34 point-of-care screening enabling rapid and reproducible classification of clinically

35 relevant enterococci. 
Enterococcus is a highly significant genus of bacteria, which causes important

38 clinical infections including urinary tract infections (UTIs), endocarditis, meningitis,

39 catheter-related infections, bacteremia, wound infections, pelvic and intra-abdominal

40 infections amongst others. Some of these Gram-positive cocci were originally

41 classified as Streptococcus spp. until genomic analysis by Schleifer and Kilpper-

42 Balz in 1984 demonstrated the requirement for a separate genus classification (1).

43 This well-known genus is part of the normal intestinal microflora of humans and

44 other animals (2). Enterococcus are also part of the lactic acid bacteria (LAB) group

45 present in foods, and whilst they are able to spoil fresh meats (3), they are important

46 in ripening and development of certain foods (i.e. dairy products), as well as being

47 used as probiotics in humans (4).

48 The majority of human clinical isolates of enterococci belong to two species,

49 Enterococcus faecalis and Enterococcus faecium (5). In addition to their prevalence

50 and pathogenicity, another very important factor associated with enterococcus is the

51 high level of antimicrobial resistance, particularly resistance to glycopeptide

52 antibiotics (such as vancomycin, teicoplanin and telavancin); resistant strains are

53 referred to as GRE (glycopeptide-resistant enterococci) $(6,7)$.

54 There is a constant requirement to develop analytical methods for the 55 discrimination of bacteria, which can be used in clinical diagnostics and food quality 56 control. These methods should ideally be rapid, reproducible, easy to use and 57 automated, in addition to having high resolution and sensitivity (8). Over a decade 58 ago, it was common to use methods, such as polymerase chain reaction (PCR) for 59 identification of specific DNA sequences and recognition by antibodies via enzyme60 linked immunosorbent assay (ELISA), to characterize bacteria. Although these 
61 techniques are sensitive and specific, they are time-consuming and their use is

62 limited by the complexity of preparation procedures and the requirement for specific

63 primers and antibodies (9-12). Nowadays, modern analytical techniques, such as

64 matrix-assisted laser desorption/ionization time-of-flight mass spectrometry

65 (MALDI-TOF-MS) (13-16), Fourier transform infrared (FT-IR) spectroscopy (17-

66 21) and Raman spectroscopy (22-24) are also used for the characterization of

67 bacteria. High dimensional and information rich datasets are produced from these

68 techniques, which has also directly led to the requirement of robust and reliable

69 chemometric methods to assist with data deconvolution and in-depth analysis (25).

70 This saw the introduction, acceptance and use of chemometrics, such as discriminant

71 function analysis (DFA) (22) and hierarchical cluster analyses (HCA) (26-28).

72 Previously, MALDI-TOF-MS has shown promising results for bacterial 73 characterization (13). FT-IR and Raman spectroscopy complement each other for 74 bacterial classification; both are robust metabolic fingerprinting techniques and need 75 little sample preparation $(29,30)$. FT-IR spectroscopy is used by many researchers 76 since it is not only rapid but also offers a high-throughput and non-destructive 77 method, allowing the analysis of intact bacteria and producing unique, reproducible 78 and distinct biochemical fingerprints (31). Raman spectroscopy shares similar 79 advantages to FT-IR spectroscopy and also has the additional advantage of water 80 being a very weak Raman scatter (32) so that samples do not need to be dried.

81 Here, the aim was to use these three distinct phenotypic approaches (namely

82 MALDI-TOF-MS, FT-IR and Raman spectroscopies) in combination with rigorous 83 chemometric analysis of the resultant datasets to classify 35 clinically relevant 84 isolates of enterococci, which had been previously typed by pulsed-field gel 85 electrophoresis (PFGE). This was carried out in order to compare the results from, 
86 and determine the efficiency of, these analytical techniques for the rapid

87 differentiation of E. faecium strains. In future, this may allow clinical diagnostic

88 laboratories to analyze multiple bacterial samples rapidly for infection control

89 purposes in point-of-care setting within hospitals, clinics, or GP surgeries which

90 would significantly accelerate diagnosis, and potentially ensure that the correct

91 antimicrobial therapies were used if required, and eliminate the delay associated with

92 sending strains to reference laboratories when analyzing patient samples.

95 MATERIALS AND METHODS

96 General chemicals. Trifluoroacetic acid (TFA), HPLC grade water, acetonitrile, 97 sinapinic acid (SA), $\alpha$-cyano-4-hydroxycinnamic acid (CHCA), and ferulic acid 98 (FA) were purchased from Sigma-Aldrich (Dorset, UK).

99 Enterococci strains. Isolates were from faecal samples from patients in a surgical 100 ward in a hospital in Belfast, UK and were collected following an increase in 101 enterococcal infections on the ward. They were identified as E. faecium by a 102 VITEK ${ }^{\circledR}$ system (bioMérieux) and their identity confirmed by MALDI-ToF analysis 103 using a Bruker microflex instrument. The 35 isolates were typed using pulsed-field 104 gel electrophoresis (PFGE) of SmaI-digested genomic DNAby Public Health 105 England's National Reference Laboratory as described previously (33). Table S1 106 summarizes information on the 35 clinical isolates, which were classified into 12 107 groups (12 PFGE-defined types) named: EC04, EC09, EC10, EC13, EC14, EC15, 108 EC19, EC20, UNI 156, UNI 178, UNI 191 and UNI 214, where 'UNI' types 109 describe isolates that were unique within the set.

\section{Bacterial isolates.}


111 The samples analyzed by the three techniques (viz. MALDI-TOF-MS, FT-IR and

112 Raman) were collected from the same flask to avoid any variations between different 113 preparations that may affect results obtained using the different anlaytical platforms.

114 First, enterococci were cultured on nutrient agar (NA) plates for $24 \mathrm{~h}$ at $37^{\circ} \mathrm{C}$. A 115 single colony from the agar culture was used to inoculate $50 \mathrm{~mL}$ of Lysogeny broth 116 (LB) in a $250 \mathrm{~mL}$ flask which was incubated overnight at $37^{\circ} \mathrm{C}$ with shaking at 200 $117 \mathrm{rpm}$. This was followed by measuring the optical density (OD) at $600 \mathrm{~nm}$ using a 118 Biomate 5 spectrophotometer (Thermo, Hemel Hempstead, UK) for each isolate. 119 The volume of analyzed bacterial suspension was then normalized to account for 120 variation in cell biomass in the different replicate cultures (4 biological replicates 121 were prepared for each isolate) and used to inoculate a fresh flask of broth, which 122 was incubated at $37^{\circ} \mathrm{C}$ for $11 \mathrm{~h}$ (when the bacteria reached the stationary phase). 123 Then, $10 \mathrm{~mL}$ from each flask was collected and centrifuged at $4800 \mathrm{~g}$ for $10 \mathrm{~min}$ and 124 the pellet washed three times with sterile deionized water. Figure S1 illustrates the 125 preparation process.

126 For vibrational spectroscopic analysis, the collected pellets were suspended in 127 suitable volumes of saline $(0.9 \%(\mathrm{w} / \mathrm{v}) \mathrm{NaCl})$ depending on the OD (all isolates had 128 approximately the same cell density). Then, $15 \mu \mathrm{L}$ was spotted onto a silicon plate 129 (Bruker Ltd., Coventry, UK) and was allowed to dry at $40^{\circ} \mathrm{C}$ for $45 \mathrm{~min}$ before 130 analysis with FT-IR spectroscopy. For Raman spectroscopy, $4 \mu \mathrm{L}$ of each sample 131 was spotted onto a stainless steel plate and then allowed to dry at $40^{\circ} \mathrm{C}$ for $45 \mathrm{~min}$.

132 For MALDI-TOF-MS, three different matrices were tested to find the most 133 compatible matrix with enterococci; these matrices were: FA, SA and CHCA. In 134 addition, 3 different deposition methods (sample-matrix) were tested as described 135 previously (16) to find the best method for depositing the samples: mix, overlay and 
136 underlay (data not shown). SA matrix and the mix deposition method were found to 137 be the optimal combination for MALDI-TOF-MS analysis for these samples. On the 138 day of analysis of the samples, the biomass was suspended in $1000 \mu \mathrm{L}$ of $2 \% \mathrm{TFA}$ 139 then vortexed for $3 \mathrm{~min}$. An equal volume of $10 \mu \mathrm{L}$ of bacterial suspension and 140 matrix were vortexed for $2 \mathrm{~s}$ and $2 \mu \mathrm{L}$ of this mixture spotted onto a MALDI 141 stainless steel plate and allowed to dry at ambient temperature.

142 Fourier transform infrared (FT-IR) spectroscopy. FT-IR spectroscopy plate 143 (Bruker Ltd., Coventry, UK) which contained 96 locations/spots was washed using $1445 \%$ sodium dodecyl sulfate (SDS) solution. This was followed by washing the plate 145 using deionized water and allowing it to dry at room temperature (34). High146 throughput screening (HTS) was carried out using a Bruker Equinox 55 FT-IR 147 spectrometer. The HTX ${ }^{\mathrm{TM}}$ module described by Winder et al. (35) was used with this 148 instrument. Transmission mode was used to analyze the dried biomass to produce 149 FT-IR spectra. The parameters used for FT-IR analysis included the following: 150 spectra were collected in the wavenumber range between 4000 and $600 \mathrm{~cm}^{-1}$, 151 resolution was $4 \mathrm{~cm}^{-1}$ and each spectrum was the average of 64 co-adds. Spectral 152 acquisition and subtracting the background were achieved using Opus software 153 (Bruker Ltd.). Four biological replicates, each in four analytical replicates, were 154 analyzed and analysis was performed in three machine runs, resulting in 1680 FT-IR 155 spectra.

156 Raman Spectroscopy. This was carried out using a confocal Raman system (inVia, 157 Renishaw plc., Wotton-Under-Edge, UK) coupled with a $785 \mathrm{~nm}$ wavelength laser. 158 A power intensity of $\sim 30 \mathrm{~mW}$ was applied on the samples at an exposure time of 15920 s. Four biological replicates and seven different locations within each sample spot 160 were analyzed, resulting in a total of 980 Raman spectra. 
161 MALDI-TOF-MS. The enterococci isolates were analyzed using an AXIMA162 Confidence MALDI-TOF-MS (Shimadzu Biotech, Manchester, UK), equipped with 163 a nitrogen pulsed UV laser with a wavelength of $337 \mathrm{~nm}$. The parameters of this 164 device were set as follows: $90 \mathrm{mV}$ laser power, 91 acquired profiles with each 165 profile containing 20 shots, linear TOF, positive ionization mode, and mass-to166 charge $(\mathrm{m} / \mathrm{z})$ range of $1,000-18,000$. The spectra were collected using a circular 167 raster pattern. The MALDI-TOF-MS device was calibrated using a protein mixture: 168 insulin $(5,735 \mathrm{Da})$, cytochrome c (12,362 Da), and apomyoglobin (16,952 Da) 169 (Sigma-Aldrich). Each of 4 biological replicates from the 35 isolates was analyzed in

170 four technical replicates on four different days; this led to the generation of a total of 171560 MALDI-TOF-MS spectra (35 isolates $\times 4$ biological replicates $\times 4$ analytical 172 replicates).

\section{Data analysis}

174 Data pre-processing. Opus software was used to export FT-IR data into ASCII 175 format; the data were then transferred into MATLAB 2012a (The Mathworks Inc., 176 MA, US). All FT-IR spectra were baseline corrected using standard normal variate 177 (SNV) to remove any light scattering effect. The three analytical replicates were then 178 averaged to reduce the number of redundant samples. Due to the large number of 179 samples, 8 separate (96 spot silicon) sampling plates were used; therefore, it was 180 necessary to correct for the subtle differences in signals from different silicon plates. 181 This was achieved by using a piece-wise direct standardization (PDS) model (36). 182 The PDS model was built on two different 'reference' isolates which were spotted on 183 every plate. The pre-processed FT-IR spectra were then subjected to multivariate 184 analysis (MVA, see below). Raman spectra were also normalized using standard 185 normal variate (SNV) and then subjected to MVA. 
186 MALDI-TOF-MS data were pre-processed as follows: (i) the baseline was 187 corrected using asymmetric least squares (AsLS) (37), and (ii) spectra were 188 normalized by dividing each individual baseline corrected spectrum by the square 189 root of the sum of squares of the spectrum (38). The pre-processed MALDI-TOF190 MS data were subjected to the same data analysis flow as Raman and FT-IR spectral 191 data.

192 Multivariate data analysis. A flowchart of multivariate data analysis is provided in 193 Figure 1. For all three datasets, two types of classification were performed: one at 194 the strain level (i.e. 12 classes) defined by PFGE, and the other at the isolate level 195 (i.e. 35 classes, one for each isolate).

196 For cluster analyses, principal components-discriminant function analysis (PC197 DFA) (39-41) was applied to reduce the dimensionality of the data and discriminate 198 samples from the designated classes. The PC-DFA scores of each class were then 199 averaged and subjected to hierarchical cluster analysis (HCA) (42). Dendrograms 200 from each analysis were generated to illustrate the relative relatedness of these 201 bacteria.

202 Partial least squares-discriminant analysis (PLS-DA) (43), with 1,000 203 bootstrapping validations (44), was also applied to obtain a validated supervised 204 classification model for discriminating different strains or isolates. In each 205 bootstrapping process, the data were randomly split into two different sets: a 206 training set and a test set. A PLS-DA model was trained on the training set and then 207 applied to the test set to predict the class membership of the samples in the test set. 208 This process was repeated 1,000 times and the results were recorded and averaged to 209 produce a $c \times c$ confusion matrix ( $c$ is the number of designated classes, either 12 210 (strains) or 35 (isolates)), in which the element at the $i^{\text {th }}$ row, $j^{\text {th }}$ column is the 
211 percentage of samples in class $i$ being predicted as class $j$ on average. In order to

212 assess the statistical significance of the predictive performance of the PLS-DA

213 models, a corresponding permutation test within each bootstrapping resampling was

214 also performed. This means that in addition to building the PLS-DA model using the

215 known class membership, another model (called the 'null' model) was also built

216 using a randomly permuted class membership. The results of the null model were

217 also recorded and from this the null distribution was obtained. An empirical $p$-value

218 was calculated by counting the number of cases where the null model had obtained

219 better predictive accuracy than the real model and dividing the obtained number by

220 the total number of bootstrapping resampling (i.e. 1,000 in this study).

221 Finally, similarities between the three different datasets (FT-IR spectroscopy,

222 Raman spectroscopy and MALDI-TOF-MS data) were measured using Procrustes

223 analysis (45). Procrustes analysis is an excellent approach for assessing the

224 differences and similarities between different ordination space from cluster analyses

225 and has been used previously for the assessment of different analytical techniques

226 (46). The distances were calculated based on the averaged PC-DFA scores for the

227 biological replicates.

229 RESULTS AND DISCUSSION

230 Table $\mathrm{S} 1$ shows all 35 isolates belonging to 12 strains (PFGE-defined 12 types)

231 including: EC04, EC09, EC10, EC13, EC14, EC15, EC19, EC20 UNI 156, UNI 178,

232 UNI 191 and UNI 214. These strains were previously confirmed to belong to $E$.

233 faecium using a VITEK $^{\circledR}$ system and by MALDI-ToF analysis using a Bruker

234 Microflex system (data not shown). The PFGE results (Fig. S2) were compared to 
235 results obtained in this study using FT-IR spectroscopy (17, 30, 46-49), Raman 236 spectroscopy $(25,30,50,51)$ and MALDI-TOF-MS $(13,14,16,52-54)$. We believe

237 that these analytical techniques in combination with chemometrics offer an

238 improvement in the classification of bacteria due to their higher biochemical 239 resolution.

\section{Classification using FT-IR spectroscopy.}

241 In this study, four biological replicates of bacterial isolates were analyzed in four 242 analytical replicates and analysis was performed in three machine runs, resulting in a 243 total of 1680 FT-IR spectra. The three machine replicate measurements were 244 performed in order to evaluate the reproducibility of the FT-IR technique. Typical 245 spectra based on four biological replicates of representatives of 12 strains from 246 enterococcus (EC04, EC09, EC10, EC13, EC14, EC15, EC19, EC20, UNI 156, UNI

247 178, UNI 191 and UNI 214) are provided in Figure S3A. The infrared spectra 248 contain different distinct regions that can be used to characterize bacterial samples. 249 These have been well documented previously and include: wavenumbers around $250 \quad 3400-2850 \mathrm{~cm}^{-1}$ corresponding to fatty acids, at $1705-1454 \mathrm{~cm}^{-1}$ related to amide I 251 and II regions attributed to peptides and proteins, and around 1085-1052 $\mathrm{cm}^{-1}$ 252 corresponding to polysaccharides $(19,55,56)$.

253 Discrimination between the strains based on visual inspection of the spectra was 254 difficult (17) because these strains are very similar phenotypically. Therefore, in 255 order to develop a classification model to distinguish between bacterial samples 256 based on similarities in the spectral data, multivariate analysis was used to reduce the 257 high dimensionality of the data. First, PC-DFA was applied using 40 principal 258 components (PC) to the 12 strains (i.e. 12 classes) and 35 isolates (i.e. 35 classes) 259 using the pre-processed FT-IR spectra (Fig. 2A and 3A, respectively). Figure 2A 
shows a clear separation between the 12 strains, displaying 4 main clusters; Cluster 1

261 is a single-member cluster (SMC) containing only (EC10), Cluster 2 includes (EC20

262 and UNI 156), Cluster 3 (UNI 191, EC04 and EC15) and Cluster 4 formed a large

263 group and is a combination of (EC13, EC19, EC14, EC09, UNI 214 and UNI 178).

264 Each cluster is represented by a different color in the figure. As described above,

265 HCA was undertaken using spectral data in order to simplify the DFA plot and to

266 illustrate the related strains. Cluster analysis was based on averaged DFA scores (12

267 classes/strains), using Ward's linkage as shown in Figure 2B. Clusters seen in Figure

$2682 \mathrm{~A}$ are reflected in the HCA dendrogram plot (Fig. 2B).

269 PC-DFA was subsequently performed for all the 35 isolates and the results are

270 provided in Figure 3. Clear separation between all 35 isolates was observed despite

271 the fact that there were a much higher number of classes to be separated than the

272 number of strains. For example, clear separation was observed between the two

273 representatives of EC10 (139 and 151). Furthermore, results generated using PFGE

274 correlated well with FT-IR spectroscopic data. For example, the UNI 156 and

275 UNI 178 were seen as unique by both techniques. In addition, the three EC20

276 isolates (192, 198 and 204) and EC19 isolates (173, 174 and 175) clustered together

277 and were not differentiated using FT-IR spectroscopy, which was also observed in

278 the PFGE results, where the bands were quite similar (Fig. 3B). This implies that the

279 isolates within each of these groups are highly similar to each other phenotypically

280 and genetically. Finally, two more clusters were observed, with one cluster

281 containing all the EC04, EC15 and UNI 191 strains and the remainder of the isolates

282 forming another cluster.

283 The PLS-DA classification using FT-IR spectral data achieved an average correct

284 classification rate (CCR) of $89.4 \%$ at the strain level and $54.3 \%$ at the isolate level, 
285 both with an empirical $p$-value of $<0.001$, i.e. not a single case where the null model 286 obtained better results, indicating that the predictive accuracies were highly

287 significant. The null distributions are provided in Figure S4A and B at the two 288 levels.

289 The confusion matrices of strains and isolates classification are presented in 290 Table 1 and Table S3, respectively. Most of the 12 strains showed high prediction 291 accuracies, for example EC04, EC10, EC13 and EC20 had accuracies of 89.9\%, $29299.7 \%, 99.8 \%$ and 99.2\%, respectively. However, EC14 and UNI 214 had lower 293 prediction accuracies of $47.3 \%$ and $58.9 \%$, respectively. The confusion matrix 294 showed that there was a certain level of overlap between (EC14 and EC09) and (UNI 295214 and EC19).

297 Furthermore, in-depth analysis of the confusion matrix (Fig. 4) showed that 298 classification of unique strains was generally in line with PFGE results. In Figure 4, 299 high percentage class membership assignments are represented by warm colors (e.g. 300 red), indicating agreement between predicted classes and known classes. It is also 301 interesting to see that representatives from EC19 and EC20 formed two "squares" of 302 "tiles" on the diagonal line, in which the colors were similar to each other. Results 303 from Figure 4 suggest that the PLS-DA model was not able to differentiate the 304 isolates within EC19 and EC20, yet another observation that is consistent with PFGE 305 results. On the other hand, all representatives of EC04 and EC09 (160 and 133) were 306 unique in the FT-IR spectroscopy profile using the PLS-DA model but had visually 307 similar PFGE profiles. This is most likely due to PFGE providing genetic 308 information $(57,58)$ while FT-IR spectroscopy describes phenotypes $(27,59)$. This 309 implies that isolates from EC19 and EC20 are highly conserved phenotypically, 
310 whereas those from EC04 and EC09 are not, and such subtle differences in

311 phenotypes were detected by FT-IR spectroscopy. Our observations showed that FT-

312 IR spectroscopy appears to be a very promising analytical approach for

313 discrimination of enterococci at different levels. In line with the results presented in

314 this study, work carried out by Guibet et al. showed that clear discrimination and

315 classification of enterococci strains can be achieved using FT-IR spectroscopy (60, 316 61).

317 Classification using Raman spectroscopy. In addition to the FT-IR spectroscopy 318 technique used in this study, Raman spectroscopy was used as a complementary 319 technique $(17,61-63)$. As expected, the two techniques generated different spectra. 320 These two approaches are complementary due to the selection rules, whereby 321 infrared causes a change in the net dipole moment in a particular functional group, 322 induced by molecular vibrations, whereas Raman causes a change in the polarization 323 of bonds within a molecule. Therefore, bonds within a molecule are generally 324 infrared or Raman active with the result being that the two techniques can provide 325 complementary (bio) chemical information $(29,64)$.

326 Raman spectra of the 12 E. faecium strains are shown in Figure S3B. Raman 327 spectra for these types appeared almost indistinguishable and no differences were 328 detected on visual inspection. Moreover, some specific peaks which were identified 329 in these spectra included: peaks at around $722 \mathrm{~cm}^{-1}, 783 \mathrm{~cm}^{-1}, 854 \mathrm{~cm}^{-1}, 1004 \mathrm{~cm}^{-1}$, $3301098 \mathrm{~cm}^{-1}, 1334 \mathrm{~cm}^{-1}, 1451 \mathrm{~cm}^{-1}$ and $1664 \mathrm{~cm}^{-1}$, which correspond to adenine, 331 cytosine/uracil, tyrosine, phenylalanine, phosphate, guanine, protein and amide I, 332 respectively $(65-67)$.

333 PC-DFA scores plot of pre-processed Raman spectra for the 12 PFGE-defined 334 types is shown in Figure S5A. The figure shows classification results similar to those 
335 seen with FT-IR spectroscopy data. There was an obvious overlap between the two 336 spectroscopic techniques, especially with representatives of EC10. However, EC20

337 overlapped with UNI 156 in FT-IR spectroscopy data, whereas EC20 was closer to

338 UNI 178 based on Raman spectroscopy data. These observations can be seen in the 339 HCA dendrogram based on Raman data (Fig. S5B), which was quite similar to the 340 HCA results generated from FT-IR data. Looking back at the dendrogram in 341 Figure S2 based on PFGE data, visual inspection showed that there were some 342 similarities between results generated via spectroscopic techniques and those based 343 on PFGE; for example, EC04 and EC15 were shown to overlap in both sets of results 344 (Fig. S2).

345 As with FT-IR data, Raman spectroscopy data on the 35 isolates were also 346 analyzed using to PC-DFA and HCA (Fig. S6A and B, respectively). The results 347 suggested that Raman spectroscopy was also successful in discriminating the two 348 representatives of EC10 (139 and 151), which was also the case using FT-IR 349 analysis (Fig. 3). Furthermore, in order to ensure the classification is robust, the data 350 were analyzed using a heat map based on PLS-DA (Fig. S6C). The results suggested 351 that all the isolates indicated as unique (UNI) by PFGE were also unique in the PLS352 DA model generated using Raman spectroscopy data.

353 In addition, chemometric-based identification was carried out using PLS-DA at 354 both the strain and isolate levels and the predictive accuracies were calculated based 355 on 1,000 bootstrapping resampling using Raman spectral data. The null distribution 356 was obtained (Fig. S4C and D) at both the strain (12 classes) and isolate levels (35 357 classes) resulting in average CCR of $69.3 \%(p<0.001)$ and $21.1 \%(p<0.001)$, 358 respectively. The CCR from FT-IR data was higher at both levels compared to 359 Raman data possibly due to the higher reproducibility of FT-IR data. Confusion 
matrices were also generated at both the strain level (Table S2A) and the isolate

361 level (data not shown); these results suggested that Raman spectroscopy can also be

362 used as a robust technique for bacterial discrimination. In-depth analysis showed that

363 Raman spectroscopy generated around 70\% prediction accuracy at the strain level

364 which is lower than that of FT-IR spectroscopy (nearly 90\%). This is most likely due

365 to the low concentration of cells used for analysis: the infrared interrogation beam

366 used was $c a .1 \mathrm{~mm}$ and passes completely through the dried bacterial film; while the

367 Raman microscope delivers a highly focussed laser beam with an interrogation

368 volume of $\sim 1 \mathrm{pL}$ and therefore measures very few bacteria. To overcome this

369 limitation with Raman, bacteria can be analyzed directly from the agar plates or

370 surface-enhanced Raman spectroscopy (SERS) as an alternative technique (68-70),

371 but this is an area for future study.

372 Classification using MALDI-TOF-MS. As described in the Materials and Methods

373 section, four biological replicates were analyzed in four analytical replicates for each

374 bacterial strain, resulting in 560 MALDI-TOF-MS spectra; both the biological and

375 technical replicates clustered closely together ensuring good bioanalytical

376 reproducibility (data not shown). The spectra for the 35 enterococci isolates were

377 pre-processed before data analysis. The typical pre-processed positive ion mode

378 MALDI-TOF-MS spectra for all 12 Enterococcus strains (EC04, EC09, EC10,

379 EC13, EC14, EC15, EC19, EC20, UNI 156, UNI 178, UNI 191 and UNI 214) are

380 provided in Figure S3C. In general, the MALDI-TOF-MS spectra were of high

381 quality with high signal-to-noise ratios in the acquisition $\mathrm{m} / \mathrm{z}$ range $1,000-18,000$ and

382 a high number of peaks for each studied strain were detected. There are many factors

383 that can affect MALDI-TOF-MS results and some of these can differ from lab to

384 another, such as the type of medium used (71), sample handling, type of matrix (72), 
385 sample deposition method (73), solvents, instrument settings $(74,75)$ and the type of 386 data analysis chosen $(41,76)$. These can inadvertently affect MALDI-TOF-MS 387 results and subsequent PC-DFA and HCA.

MALDI-TOF-MS spectra are not readily interpretable from the 35 isolates as 389 they are similar phenotypically and MALDI-TOF-MS spectra show only two 390 dimensions $(\mathrm{m} / \mathrm{z} \times$ intensity). Therefore, as is the case for the vibrational 391 spectroscopy techniques, robust multivariate analysis methods were employed for 392 this purpose. The results of PC-DFA using 12 classes (12 strains) in a three393 dimensional plot of DF1 vs DF2 vs DF3 and a two-dimensional plot of DF2 vs DF3 394 are shown in Figure S7A and B, respectively. Four main clusters were observed in 395 the PC-DFA plots; SMC (Cluster) 1 contains only UNI 178; Cluster 2 contains 396 EC20; Cluster 3 consists of EC04, EC10, EC15 and UNI 191; and Cluster 4 formed 397 a large group of (EC13, EC19, EC14, EC09, UNI 214 and UNI 156). Results from 398 the HCA dendrogram (Fig. S7C) confirmed the separation between the 12 classes 399 (i.e. 12 strains). This indicated that UNI 178 is phenotypically very different from 400 the other strains based on MALDI-TOF-MS data.

PC-DFA was also applied to data from the 35 isolates; the results showed 402 that isolates number 160 and 219 (both from EC09) were very different from the 403 other isolates. Therefore, another PC-DFA was carried out with these two outliers 404 removed and the HCA results are shown in Figure S8D. It appears that all 405 representatives of EC20 (204, 198 and 192) overlap with each other, which was also 406 observed in FT-IR and Raman spectroscopy data, with the exception that isolate 192 407 slightly differed from the other two representatives (204 and 198) in the HCA 408 dendrogram when using Raman data (Fig. S6B). However, analysis by PFGE 
showed that isolates 192 and 198 clustered more closely with each other than with 410 isolate 204.

Furthermore, PLS-DA model applied to MALDI-TOF-MS data achieved an 412 average CCR of $78.2 \%(p<0.001)$ and $35.7 \%(p<0.001)$ for the 12 (strains) and 35 413 (isolates) classes, respectively. When PLS-DA was undertaken with 33 isolates (with 414 isolates 160 and 219 removed), the average CCR for the isolates increased to $41553.95 \%(p<0.001)$. The prediction accuracies for the 12 classes are shown in Table 416 S2B and those for the 35 classes (isolates) are shown in Table S4. Table S2B shows 417 that discrimination between most of the strains (12 classes) using MALDI-TOF-MS 418 data achieved high correct classification rates, except for EC14 and UNI 191, which 419 had rather low classification rates. Confusion matrices for the 35 classes and the 33 420 classes (160 and 219 isolates removed) are shown in Figure S8A and C, respectively.

421 From these matrices, it can be seen that all the isolates identified by the reference 422 laboratory as unique (UNI), which included isolates 156, 178, 191 and 214, were 423 also classified as unique based on MALDI-TOF-MS data. Moreover, EC20 and 424 EC19 were assigned the same classification in PFGE typing, and this was in 425 agreement with MALDI-TOF-MS, FT-IR spectroscopy and Raman spectroscopy 426 data. In addition, based on MALDI-TOF-MS data (Fig.S8A and C), representatives 427 of EC13 (152, 154 and 155) belonged to the same cluster, and isolates 177 from 428 EC13 was significantly different from the remaining EC13 strains; this was also 429 observed in FT-IR and PFGE data. Looking back at Figure S8C, it can be seen that 430 all the strains from EC04 were unique in MALDI-TOF-MS and FT-IR profiles when 431 using PLS-DA modelling.

432 Procrustes distance test of the three analytical techniques. Analytical techniques 433 such as FT-IR spectroscopy, Raman spectroscopy and MALDI-TOF-MS are 
434 currently used in clinical research studies worldwide and many reports have been 435 published showing advantages of using such techniques $(24,54,77,78)$. Kirschner 436 et al. (61) demonstrated accurate identification and classification of 18 strains from 6

437 different species belonging to enterococci using vibrational spectroscopic techniques 438 in combination with chemometrics. This study suggested that FT-IR and Raman 439 spectroscopies can offer potential alternatives to the conventional typing tests due to 440 their speed and ease of use. Oliveira et al. (51) showed that Raman spectroscopy, in 441 combination with a chemometric algorithm, can be used to discriminate between 442 seven different colonies of Gram-positive and Gram-negative bacteria. In another 443 previous study, it was also shown that 59 clinical bacterial strains associated with 444 urinary tract infections (UTIs) could be identified using FT-IR and Raman 445 spectroscopy (17). As an alternative to vibrational spectroscopic techniques, 446 MALDI-TOF-MS is a relatively new technique which has shown very promising 447 results for identification in agreement with methodologies carried out in 448 microbiological laboratories, and therefore has been used for the identification and 449 classification of bacterial species $(15,79,80)$ and is appearing in many clinical 450 microbiology testing laboratories $(54,81,82)$.

451 Previous studies have generally focussed on the application of just one or two 452 analytical techniques for the classification of Enterococcus spp. However, to 453 generate complementary data and more comprehensive analysis, this study combines 454 three different analytical techniques - FT-IR spectroscopy, Raman spectroscopy and 455 MALDI-TOF-MS - to analyze whole bacterial cells. Successful classification was 456 demonstrated at the strain (i.e. 12 classes) and isolate (i.e. 35 classes) levels based on 457 data generated by the three analytical platforms. In order to assess the overall 458 information content in the spectra that has been revealed by the cluster analysis from 
459 the scores plots, Procrustes analysis was employed to assess the overall similarity

460 between the patterns detected by these three platforms. The results are presented in 461 terms of Procrustes distance (Table 2A and B), where the Procrustes distance varies

462 from 0 to 1 ; the lower the distance, the higher the similarity between the results. The 463 comparisons were made using averaged PC-DFA scores. For each dataset, there 464 were two sets of PC-DFA scores, one at the strain level (12 classes) and another for 465 isolates classification (33 classes). For each set of PC-DFA scores, the scores were 466 then averaged according to their strain label and isolate label to give two sets of 467 averaged PC-DFA scores.

468 The findings in Table 2 can be summarized as follows:

469 (i) The patterns in the PC-DFA scores at strain and isolate levels were highly 470 similar to each other for all the three analytical platforms. The Procrustes

(ii) The two vibrational spectroscopic techniques (FT-IR and Raman) generated

(iii) However, the results generated by MALDI-TOF-MS were significantly different from those generated by the two spectroscopic techniques, and the corresponding Procrustes distances were all above 0.8. Such differences can be mainly attributed to data on isolate UNI 178, which appeared to be very different to other strains in the MALDI-TOF-MS dataset. 
483 Table S5 shows a summative comparison of the 4 main clusters identified based on

484 the three analytical techniques using PC-DFA plots of the 12 E. faecium strains (12

485 classes). It can be seen from this table that despite the large Procrustes distances

486 between data generated by MALDI-TOF-MS and those generated by the other two

487 techniques, the main identified clusters patterns observed in all three datasets were

488 still largely consistent.

\section{CONCLUSIONS}

492 The results obtained from the two vibrational spectroscopic techniques 493 demonstrated that good discrimination can be achieved at both the strain and isolate 494 levels and the detected patterns from the two techniques were highly similar. In 495 addition, bacterial classification results from MALDI-TOF-MS were generally 496 consistent with these vibrational spectroscopic techniques. However, UNI 178 was 497 detected to be very different in MALDI-TOF-MS data, which differed from the other 498 two analytical techniques employed in this study.

499 The results obtained using these spectroscopic phenotyping approaches were 500 mostly consistent with previous results obtained from experiments carried out using 501 the genotypic classification method, PFGE. Some of the results differed when 502 directly comparing our analytical approach with results from the molecular approach 503 and these differences may be due to comparing phenotypic differences from whole504 organism fingerprinting with genotypic differences using PFGE.

505 In conclusion, we have assessed multiple analytical phenotypic as 506 complementary approaches to current molecular methods. All methods provided 507 excellent clustering which was in general agreement with genotypic baseline 
508 methods, as well as allowing excellent discrimination to the strain level and good 509 resolution at the sub-strain level. We believe that these three different 510 physicochemical techniques have excellent potential as high-throughput point-of511 care screening tools, and for the rapid and reproducible classification of clinically 512 relevant bacteria, such as E. faecium.

\section{ACKNOWLEDGMENTS}

514 NM thanks The Saudi Ministry of Higher Education and Princess Nora bint Abdul 515 Rahman University for funding. YX thanks Cancer Research UK (including 516 Experimental Cancer Medicine Centre award) and Wolfson Foundation, DIE and RG 517 thank BBSRC (BB/L014823/1) for support for Raman spectroscopy. 


\section{REFERENCES}

520 1. Schleifer KH, Kilpper-Bälz R. 1984. Transfer of Streptococcus faecalis and

521 Streptococcus faecium to the Genus Enterococcus nom. rev. as Enterococcus

522 faecalis comb. nov. and Enterococcus faecium comb. nov. Int. J. Syst Bacteriol.

$523 \quad 34: 31-34$

524 2. Kayser FH, Bienz KA, Eckert J. 2011. Medical microbiology. Thieme.

525 3. Hayes JR, English LL, Carter PJ, Proescholdt T, Lee KY, Wagner DD, White

526 DG. 2003. Prevalence and antimicrobial resistance of Enterococcus species straind

527 from retail meats. Appl. Environ. Microbiol. 69:7153-7160.

528 4. Franz CM, Stiles ME, Schleifer KH, Holzapfel WH. 2003. Enterococci in foods a 529 conundrum for food safety. Int. J. Food Microbiol. 88:105-122.

530 5. McCracken M, Wong A, Mitchell R, Gravel D, Conly J, Embil J, Johnston L, 531 Matlow A, Ormiston D, Simor A. 2013. Molecular epidemiology of vancomycin532 resistant enterococcal bacteraemia: results from the Canadian Nosocomial Infection 533 Surveillance Program, 1999-2009. J. Antimicrob. Chemother. 68:1505-9.

534 6. Woodford N. 1998. Glycopeptide-resistant enterococci: a decade of experience. J. $535 \quad$ Med. Microbiol. 47:849-862.

$5367 . \quad$ Arias CA, Murray BE. 2012. The rise of the Enterococcus: beyond vancomycin 537 resistance. Nature Rev. Microbiol. 10:266-278.

538 8. Altekruse S, Cohen M, Swerdlow D. 1997. Emerging foodborne diseases. Emerg. $539 \quad$ Infect. Dis. 3:285-294.

540 9. Engvall E. 1977. Quantitative enzyme immunoassay (ELISA) in microbiology. $541 \quad$ Med. Biol. 55:193.

542 10. Yolken RH. 1980. Enzyme-linked immunosorbent assay (ELISA): a practical tool 543 for rapid diagnosis of viruses and other infectious agents. Yale J. Biol. Med. and $544 \quad$ medicine 53:85. 
11. Ke D, Picard FJ, Martineau F, Ménard C, Roy PH, Ouellette M, Bergeron MG. 1999. Development of a PCR assay for rapid detection of enterococci. J. Clin. Microbiol. 37:3497-3503.

12. Reen DJ. 1994. Enzyme-linked immunosorbent assay (ELISA), p 461-466, Basic Protein and Peptide Protocols. Springer.

13. Claydon MA, Davey SN, Edwards-Jones V, Gordon DB. 1996. The rapid identification of intact microorganisms using mass spectrometry. Nat. Biotechnol. 14:1584-1586.

14. Quintela-Baluja M, Böhme K, Fernández-No IC, Morandi S, Alnakip ME, Caamaño-Antelo S, Barros-Velázquez J, Calo-Mata P. 2013. Characterization of different food-straind Enterococcus strains by MALDI-TOF mass fingerprinting. Electrophoresis 34:2240-2250.

15. Lasch P, Fleige C, Stämmler M, Layer F, Nübel U, Witte W, Werner G. 2014. Insufficient discriminatory power of MALDI-TOF mass spectrometry for typing of Enterococcus faecium and Staphylococcus aureus strains. J. Microbiol. Methods 100:58-69.

16. AlMasoud N, Xu Y, Nicolaou N, Goodacre R. 2014. Optimization of matrix assisted desorption/ionization time of flight mass spectrometry (MALDI-TOF-MS) for the characterization of Bacillus and Brevibacillus species. Anal. Chim. Acta. 840:49-57.

17. Goodacre R, Burton R, Kaderbhai N, Woodward AM, Kell DB, Rooney PJ. 1998. Rapid identification of urinary tract infection bacteria using hyperspectral whole-organism fingerprinting and artificial neural networks. Microbiology 144:1157-1170.

18. Helm D, Labischinski H, Schallehn G, Naumann D. 1991. Classification and identification of bacteria by Fourier-transform infrared spectroscopy. J. Gen. Microbiol. 137:69-79. 
572 19. Naumann D, Helm D, Labischinski H. 1991. Microbiological characterizations by

573 FT-IR spectroscopy. Nature 351:81-82.

574 20. Naumann D. 2000. Infrared spectroscopy in microbiology. Encyclopedia of $575 \quad$ analytical chemistry. John Wiley and Sons Ltd, Chichester.

576 21. Burgula Y, Khali D, Kim S, Krishnan SS, Cousin MA, Gore JP, Reuhs BL,

577 Mauer LJ. 2007. Review of mid-infrared fourier transform-infrared spectroscopy 578 applications for bacterial detection. J. Rapid Methods Autom. Microbiol. 15:146$579 \quad 175$.

580 22. López-Díez EC, Goodacre R. 2004. Characterization of microorganisms using UV 581 resonance Raman spectroscopy and chemometrics. Anal. chem. 76:585-591.

582 23. Maquelin K, Kirschner C, Choo-Smith LP, van den Braak N, Endtz HP, 583 Naumann D, Puppels GJ. 2002. Identification of medically relevant 584 microorganisms by vibrational spectroscopy. J. Microbiol. Methods 51:255-271.

585 24. Beekes M, Lasch P, Naumann D. 2007. Analytical applications of Fourier 586 transform-infrared (FT-IR) spectroscopy in microbiology and prion research. Vet. 587

588 25. Ellis DI, Cowcher DP, Ashton L, O'Hagan S, Goodacre R. 2013. Illuminating 589 disease and enlightening biomedicine: Raman spectroscopy as a diagnostic tool. $590 \quad$ Analyst 138:3871-3884.

591 26. Gutteridge CS, Valus L, Macfie HJH. 1985. 14 - Numerical Methods in the 592

595 27. Davis R, Mauer L. 2010. Fourier transform infrared (FT-IR) spectroscopy: a rapid 596 tool for detection and analysis of foodborne pathogenic bacteria. Current research, 597 technology and education topics in applied microbiology and microbial $598 \quad$ biotechnology 2:1582-1594. 
28. Dworzanski JP, Deshpande SV, Chen R, Jabbour RE, Snyder AP, Wick CH, Li L. 2006. Mass Spectrometry-Based Proteomics Combined with Bioinformatic Tools for Bacterial Classification. J. Proteome. Res. 5:76-87.

29. Goodacre R, Radovic BS, Anklam E. 2002. Progress toward the rapid nondestructive assessment of the floral origin of European honey using dispersive Raman spectroscopy. Appl. Spectrosc. 56:521-527.

30. Ellis DI, Goodacre R. 2006. Metabolic fingerprinting in disease diagnosis: biomedical applications of infrared and Raman spectroscopy. Analyst 131:875-885.

31. Argyri AA, Jarvis RM, Wedge D, Xu Y, Panagou EZ, Goodacre R, Nychas GJE. 2013. A comparison of Raman and FT-IR spectroscopy for the prediction of meat spoilage. Food Control 29:461-470.

32. Goodacre R, Timmins EM, Rooney PJ, Rowland JJ, Kell DB. 1996. Rapid identification of Streptococcus and Enterococcus species using diffuse reflectanceabsorbance Fourier transform infrared spectroscopy and artificial neural networks. FEMS Microbiol. Lett. 140:233-239.

33. Woodford N, Reynolds R, Turton J, Scott F, Sinclair A, Williams A, Livermore D. 2003. Two widely disseminated strains of Enterococcus faecalis highly resistant to gentamicin and ciprofloxacin from bacteraemias in the UK and Ireland. J Antimicrob Chemother. 52:711-714.

34. Patel SA, Currie F, Thakker N, Goodacre R. 2008. Spatial metabolic fingerprinting using FT-IR spectroscopy: investigating abiotic stresses on Micrasterias hardyi. Analyst 133:1707-1713.

35. Winder CL, Gordon SV, Dale J, Hewinson RG, Goodacre R. 2006. Metabolic fingerprints of Mycobacterium bovis cluster with molecular type: implications for genotype-phenotype links. Microbiology 152:2757-2765.

6. Wang Y, Veltkamp DJ, Kowalski BR. 1991. Multivariate instrument standardization. Anal. Chem. 63:2750-2756.

37. Eilers PHC. 2004. Parametric Time Warping. Anal. Chem. 76:404-411. 
627 38. Brereton RG. 2003. Chemometrics: data analysis for the laboratory and chemical 628 plant. John Wiley and Sons.

629 39. Manly BF. 2004. Multivariate statistical methods. a primer. CRC Press.

630 40. Harrigan GG, LaPlante RH, Cosma GN, Cockerell G, Goodacre R, Maddox

631 JF, Luyendyk JP, Ganey PE, Roth RA. 2004. Application of high-throughput Fourier-transform infrared spectroscopy in toxicology studies: contribution to a study on the development of an animal model for idiosyncratic toxicity. Toxicol. Lett. 146:197-205.

\section{Gromski PS, Muhamadali H, Ellis DI, Xu Y, Correa E, Turner ML, Goodacre}

R. 2015. A tutorial review: Metabolomics and partial least squares-discriminant analysis-a marriage of convenience or a shotgun wedding. Anal. Chim. Act. 879:10-23.

44. Efron B, Tibshirani RJ. 1994. An introduction to the bootstrap. Chapman and Hall/CRC press.

645 45. Gower JC, Dijksterhuis GB. 2004. Procrustes problems, vol 3. Oxford University 646 Press Oxford. Allwood JW, Goodacre R. 2014. Multiple metabolomics of uropathogenic E. coli reveal different information content in terms of metabolic potential compared to virulence factors. Analyst 139:4193-4199. and related cell-wall fragments as given by FTIR. Infrared Phys. 24:233-238. 

170.

657 49. Mariey L, Signolle JP, Amiel C, Travert J. 2001. Discrimination, classification,

50. Muhamadali H, Chisanga M, Subaihi A, Goodacre R. 2015. Combining Raman and FT-IR Spectroscopy with Quantitative Isotopic Labeling for Differentiation of E. coli Cells at Community and Single Cell Levels. Anal. Chem. 87:4578-4586.

52. Cramer R, Gobom J, Nordhoff E. 2005. High-throughput proteomics using matrix-assisted laser desorption/ionization mass spectrometry. Expert Rev. Proteomics. 2: 407-20

53. Ellis DI, Dunn WB, Griffin JL, Allwood JW, Goodacre R. 2007. Metabolic fingerprinting as a diagnostic tool. Analyst, 131:875-885.

4. Carbonnelle E, Mesquita C, Bille E, Day N, Dauphin B, Beretti J-L, Ferroni A, Gutmann L, Nassif X. 2011. MALDI-TOF mass spectrometry tools for bacterial identification in clinical microbiology laboratory. Clin. Biochem. 44:104-109.

5. Kim S, Reuhs BL, Mauer LJ. 2005. Use of Fourier transform infrared spectra of crude bacterial lipopolysaccharides and chemometrics for differentiation of Salmonella enterica serotypes. J. Appl. Microbiol. 99:411-417.

6. Ellis DI, Harrigan GG, Goodacre R. 2003. Metabolic fingerprinting with Fourier transform infrared spectroscopy, Metabolic Profiling: its role in biomarker discovery and gene function analysis. p 111-124, Springer. Norwell, Massachusetts, USA.

57. Turabelidze D, Kotetishvili M, Kreger A, Morris JG, Sulakvelidze A. 2000. Improved pulsed-field gel electrophoresis for typing vancomycin-resistant enterococci. J. Clin. Microbiol. 38:4242-4245. 
58. Bannerman TL, Hancock GA, Tenover FC, Miller JM. 1995. Pulsed-field gel electrophoresis as a replacement for bacteriophage typing of Staphylococcus aureus. J. Clin. Microbiol. 33:551-555.

59. Alvarez-Ordóñez A, Mouwen DJM, López M, Prieto M. 2011. Fourier transform infrared spectroscopy as a tool to characterize molecular composition and stress response in foodborne pathogenic bacteria. J. Microbiol. Meth. 84:369-378.

60. Guibet F, Amiel C, Cadot P, Cordevant C, Desmonts MH, Lange M, Marecat A, Travert J, Denis C, Mariey L. 2003. Discrimination and classification of Enterococci by Fourier transform infrared (FT-IR) spectroscopy. Vib. Spectrosc. 33:133-142.

61. Kirschner C, Maquelin K, Pina P, Thi NN, Choo-Smith L-P, Sockalingum G, Sandt C, Ami D, Orsini F, Doglia S. 2001. Classification and identification of enterococci: a comparative phenotypic, genotypic, and vibrational spectroscopic study. J. Clin. Microbiol. 39:1763-1770.

62. Van de Vossenberg J, Tervahauta H, Maquelin K, Blokker-Koopmans CH, Uytewaal-Aarts M, van der Kooij D, van Wezel AP, van der Gaag B. 2013. Identification of bacteria in drinking water with Raman spectroscopy. Anal. Meth. 5:2679-2687.

63. Ch. Schroder U, Beleites C, Assmann C, Glaser U, Hubner U, Pfister W, Fritzsche W, Popp J, Neugebauer U. 2015. Detection of vancomycin resistances in enterococci within 3 [1/2] hours. Sci. Rep. 5:8217.

64. Colthup N. 2012. Introduction to infrared and Raman spectroscopy. New York, Elsevier.

65. Uzunbajakava N, Lenferink A, Kraan Y, Willekens B, Vrensen G, Greve J, Otto C. 2003. Nonresonant Raman imaging of protein distribution in single human cells. Biopolymers 72:1-9. 
66. Maquelin K, Kirschner C, Choo-Smith L-P, van den Braak N, Endtz HP, Naumann D, Puppels G. 2002. Identification of medically relevant microorganisms by vibrational spectroscopy. J. Microbiol. Methods 51:255-271.

67. Huang WE, Li M, Jarvis RM, Goodacre R, Banwart SA. 2010. Shining Light on the Microbial World: The Application of Raman Microspectroscopy. Adv. Appl. Microbiol. 70: 931-936.

68. Cotton TM, Kim JH, Chumanov GD. 1991. Application of surface-enhanced Raman spectroscopy to biological systems. J. Raman Spectrosc. 22:729-742.

69. Nabiev I, Chourpa I, Manfait M. 1994. Applications of Raman and surfaceenhanced Raman scattering spectroscopy in medicine. J. Raman Spectrosc. 25:1323.

70. Jarvis RM, Goodacre R. 2008. Characterisation and identification of bacteria using SERS. Chem. Soc. Rev. 37:931-936.

71. Shu X, Li Y, Liang M, Yang B, Liu C, Wang Y, Shu J. 2012. Rapid lipid profiling of bacteria by online MALDI-TOF mass spectrometry. nt. J. Mass spectrom. 321-322:71-76.

72. Giebel R, Worden C, Rust SM, Kleinheinz GT, Robbins M, Sandrin TR. 2010. Chapter 6 - Microbial Fingerprinting using Matrix-Assisted Laser Desorption Ionization Time-Of-Flight Mass Spectrometry (MALDI-TOF MS): Applications and Challenges, p149-184, Advances in Applied Microbiology, vol Volume 71. Academic Press.

73. Dreisewerd K. 2003. The desorption process in MALDI. Chemical reviews 103:395426.

74. Williams TL, Andrzejewski D, Lay JO, Musser SM. 2003. Experimental factors affecting the quality and reproducibility of MALDI TOF mass spectra obtained from whole bacteria cells. J. Am. Soc. Mass Spectrom. 14:342-351.

75. Freiwald A, Sauer S. 2009. Phylogenetic classification and identification of bacteria by mass spectrometry. Nat. Protoc 4:732-742. 
76. Gromski PS, Xu Y, Correa E, Ellis DI, Turner ML, Goodacre R. 2014. A comparative investigation of modern feature selection and classification approaches for the analysis of mass spectrometry data. Anal. Chim. Act. 829:1-8.

77. De Carolis E, Posteraro B, Lass-Flörl C, Vella A, Florio AR, Torelli R, Girmenia C, Colozza C, Tortorano AM, Sanguinetti M, Fadda G. 2012. Species identification of Aspergillus, Fusarium and Mucorales with direct surface analysis by matrix-assisted laser desorption ionization time-of-flight mass spectrometry. Clin. Microbiol. Infec. 18:475-484.

78. Risch M, Radjenovic D, Han JN, Wydler M, Nydegger U, Risch L. 2010. Comparison of MALDI TOF with conventional identification of clinically relevant bacteria. Swiss Med. Wkly. 140:w13095.

79. Benagli C, Rossi V, Dolina M, Tonolla M, Petrini O. 2011. Matrix-assisted laser desorption ionization-time of flight mass spectrometry for the identification of clinically relevant bacteria. PLoS One 6:e16424.

80. Dingle TC, Butler-Wu SM. 2013. MALDI-TOF mass spectrometry for microorganism identification. Clin. Lab. Med. 33:589-609.

1. Sauer S, Kliem M. 2010. Mass spectrometry tools for the classification and identification of bacteria. Nat Rev Micro 8:74-82.

Bizzini A, Durussel C, Bille J, Greub G, Prod'hom G. 2010. Performance of matrix-assisted laser desorption ionization-time of flight mass spectrometry for identification of bacterial strains routinely isolated in a clinical microbiology laboratory. J Clin Microbiol 48:1549-1554. 


\section{FIGURE LEGENDS}

760 FIG 1. Workflow of data analysis undertaken for FT-IR spectroscopy, Raman spectroscopy

761 and MALDI-TOF-MS. The data were first pre-processed then multivariate analysis MVA

762 was applied using PC-DFA at both the (ST) strain (12 classes) and (IS) isolate (35 classes)

763 levels. This was followed by PLS-DA.

764 FIG 2. (A) Discriminant function analysis (DFA) scores plot from FT-IR data after pre-

765 processing, illustrating the relationship between the 12 enterococci. (B) Cluster analysis on 766 averaged PC-DFA scores (12 classes/strains) using Ward's linkage.

767 FIG 3. (A) PC-DFA plot from FT-IR data after pre-processing which illustrates the 768 relationship between the 35 enterococcus isolates. (B) Hierarchical cluster analysis on 769 averaged PC-DFA scores (35 classes/isolates) using Ward's linkage (right) and PFGE 770 results (left). Each isolate is represented by the same color in both the boxes around the 771 PFGE images and the FT-IR dendrogram.

772 FIG 4. PLS-DA trained on 35 classes (i.e. 35 isolates) from FT-IR spectral data. High 773 percentage class membership assignments are represented by warm colors (e.g. red) whilst 774 the cold colors (e.g. blue) represent low percentage class membership assignments. The 775 diagonal "tiles" are much warmer than off-diagonal "tiles", which indicates agreement 776 between predicted classes and known classes. 


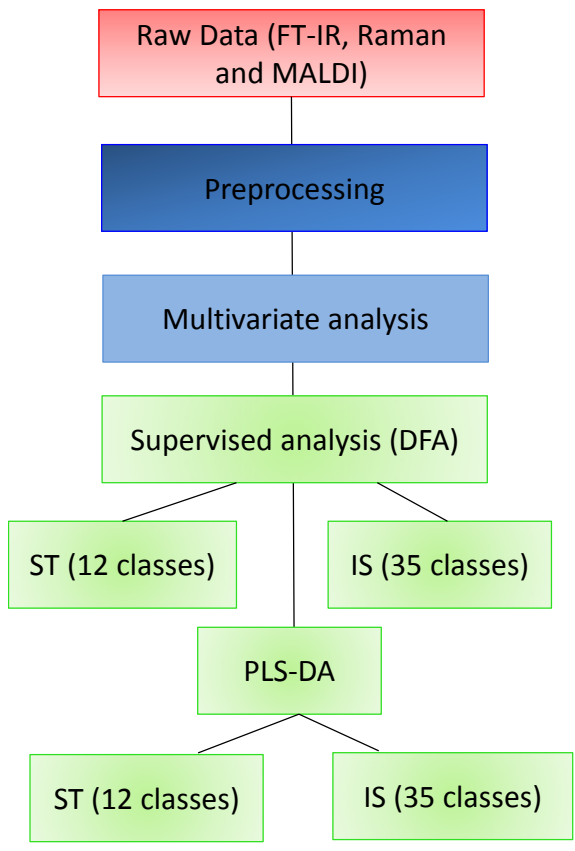

778

Figure 1 

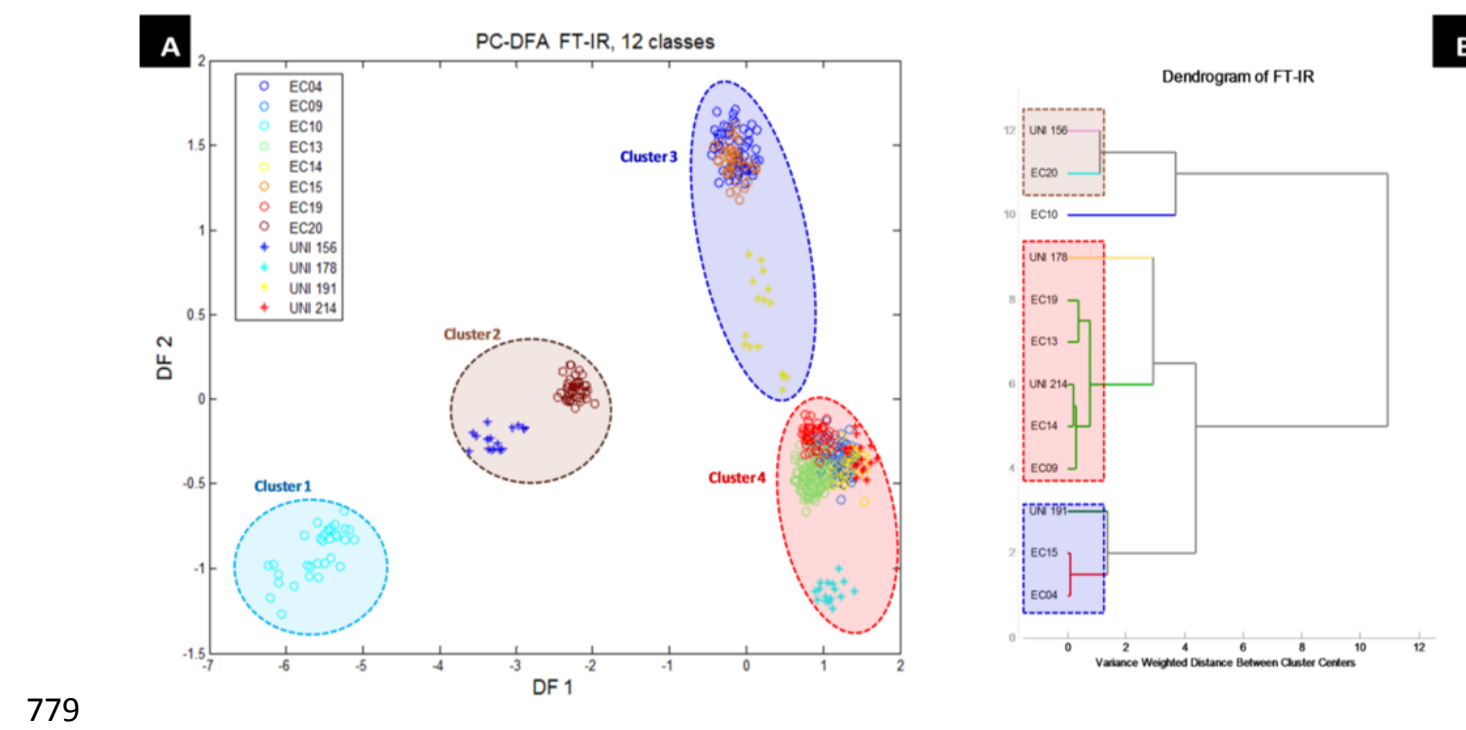

780

Figure 2 


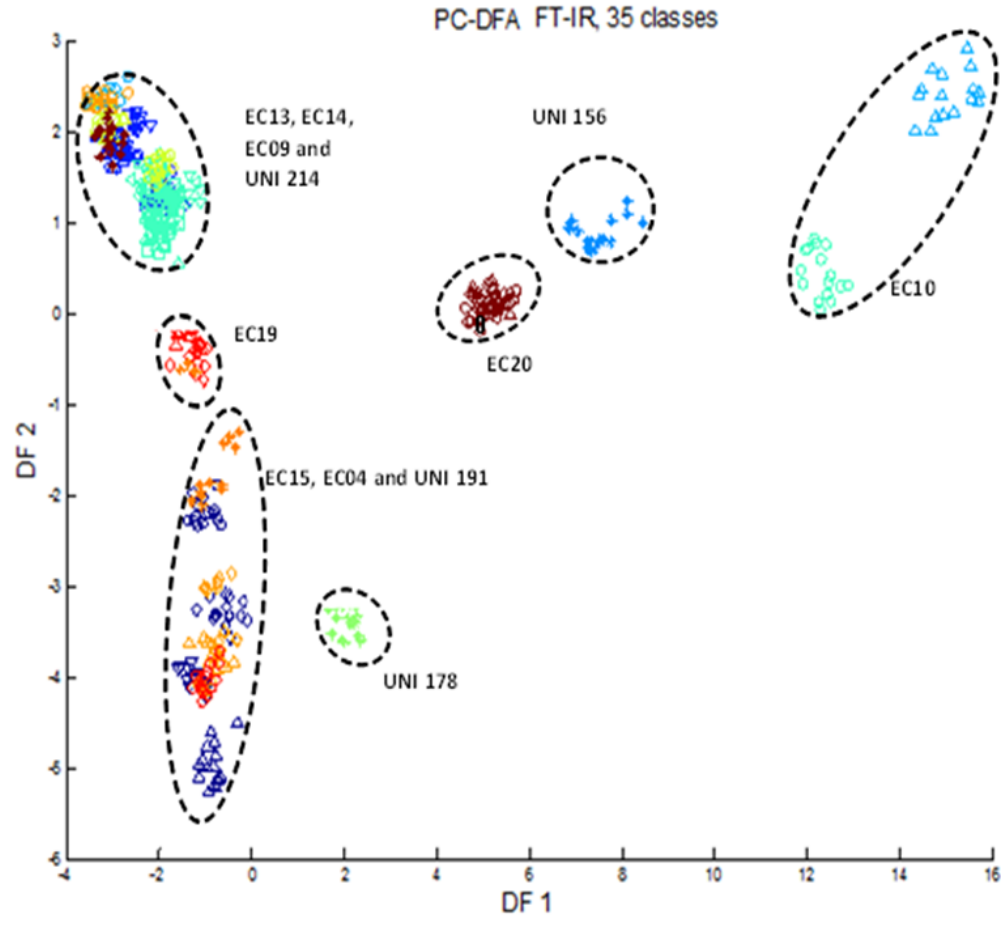

\section{PFGE}

B
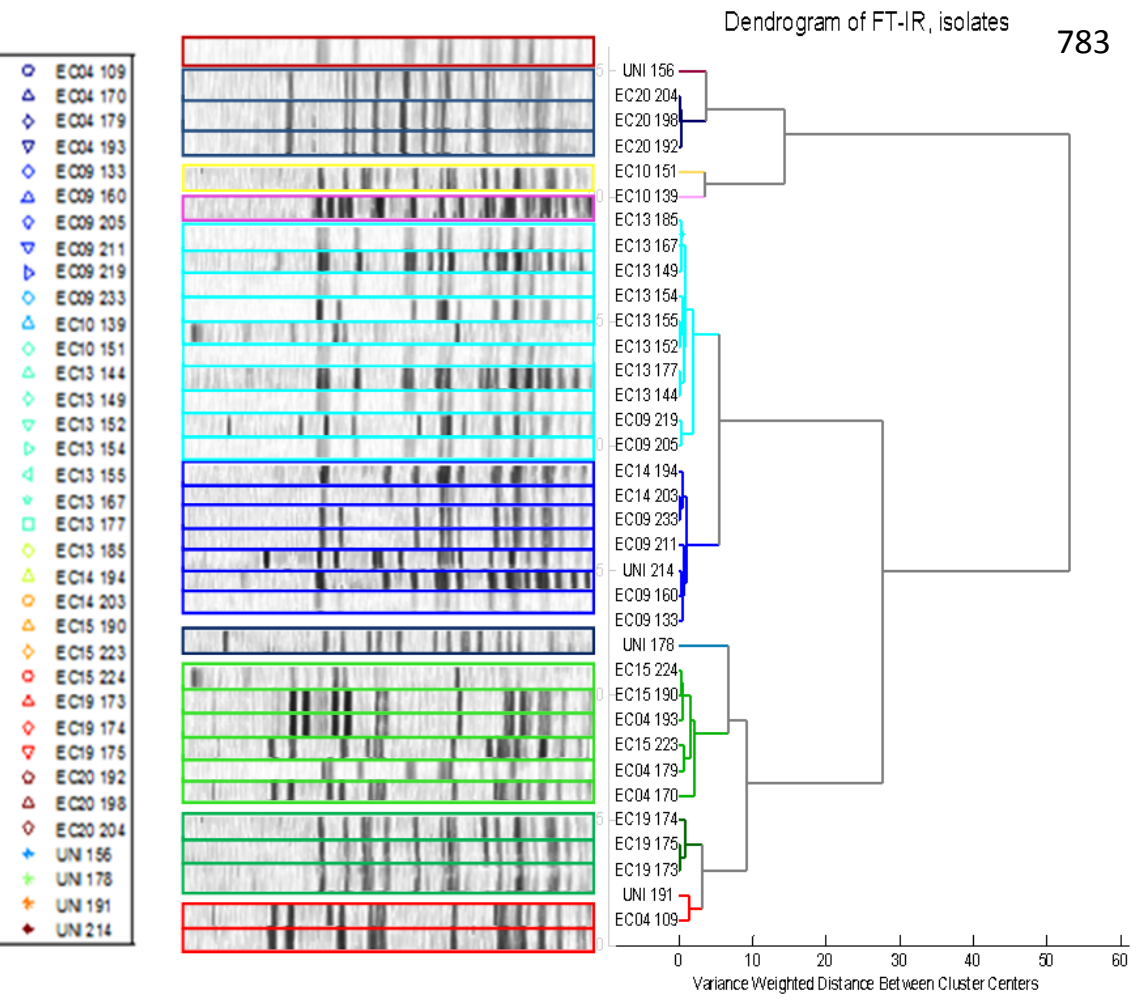

Figure 3 


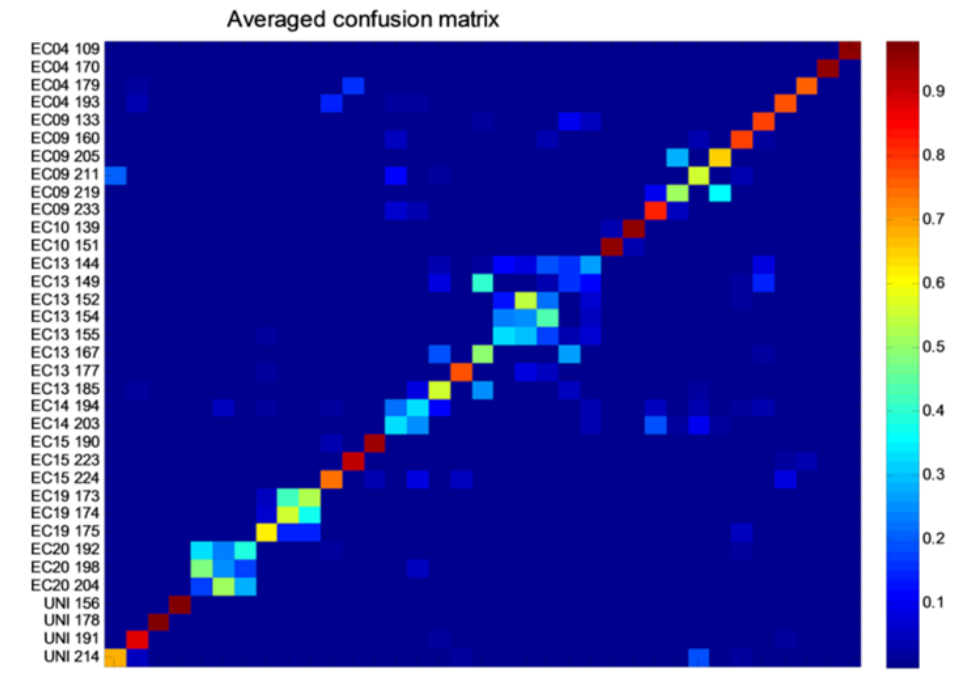

785

786

Figure 4 
787 Table 1. The prediction accuracies of the 12 enterococci strains using FT-IR spectroscopy

788 data

\begin{tabular}{|c|c|c|c|c|c|c|c|c|c|c|c|c|}
\hline $\begin{array}{l}\text { Class } \\
\text { Known/Predicted }\end{array}$ & EC04 & EC09 & EC10 & EC13 & EC14 & EC15 & EC19 & EC20 & $\begin{array}{c}\text { UNI } \\
156\end{array}$ & $\begin{array}{c}\text { UNI } \\
178\end{array}$ & $\begin{array}{c}\text { UNI } \\
191\end{array}$ & $\begin{array}{c}\text { UNI } \\
214\end{array}$ \\
\hline EC04 & $89.9 \%$ & $0.5 \%$ & $0.0 \%$ & $0.0 \%$ & $0.4 \%$ & $8.3 \%$ & $0.1 \%$ & $0.0 \%$ & $0.0 \%$ & $0.0 \%$ & $0.7 \%$ & $0.1 \%$ \\
\hline EC09 & $0.1 \%$ & $90.3 \%$ & $0.0 \%$ & $1.3 \%$ & $4.8 \%$ & $0.0 \%$ & $3.5 \%$ & $0.0 \%$ & $0.0 \%$ & $0.0 \%$ & $0.0 \%$ & $0.0 \%$ \\
\hline EC10 & $0.0 \%$ & $0.1 \%$ & $99.7 \%$ & $0.0 \%$ & $0.0 \%$ & $0.0 \%$ & $0.0 \%$ & $0.0 \%$ & $0.0 \%$ & $0.0 \%$ & $0.1 \%$ & $0.1 \%$ \\
\hline EC13 & $0.0 \%$ & $0.0 \%$ & $0.0 \%$ & $99.8 \%$ & $0.0 \%$ & $0.0 \%$ & $0.1 \%$ & $0.0 \%$ & $0.0 \%$ & $0.0 \%$ & $0.0 \%$ & $0.0 \%$ \\
\hline EC14 & $0.1 \%$ & $48.9 \%$ & $0.0 \%$ & $1.1 \%$ & $47.3 \%$ & $1.0 \%$ & $1.4 \%$ & $0.1 \%$ & $0.0 \%$ & $0.0 \%$ & $0.1 \%$ & $0.0 \%$ \\
\hline EC15 & $6.8 \%$ & $1.4 \%$ & $0.0 \%$ & $0.0 \%$ & $0.5 \%$ & $91.1 \%$ & $0.0 \%$ & $0.0 \%$ & $0.0 \%$ & $0.0 \%$ & $0.1 \%$ & $0.0 \%$ \\
\hline EC19 & $1.6 \%$ & $9.3 \%$ & $0.0 \%$ & $0.2 \%$ & $3.6 \%$ & $0.0 \%$ & $83.5 \%$ & $0.0 \%$ & $0.0 \%$ & $0.0 \%$ & $0.0 \%$ & $1.8 \%$ \\
\hline EC20 & $0.0 \%$ & $0.1 \%$ & $0.0 \%$ & $0.0 \%$ & $0.0 \%$ & $0.7 \%$ & $0.0 \%$ & $99.2 \%$ & $0.0 \%$ & $0.0 \%$ & $0.0 \%$ & $0.0 \%$ \\
\hline UNI 156 & $0.4 \%$ & $0.0 \%$ & $0.0 \%$ & $0.5 \%$ & $0.0 \%$ & $0.0 \%$ & $0.1 \%$ & $0.9 \%$ & $98.1 \%$ & $0.0 \%$ & $0.0 \%$ & $0.0 \%$ \\
\hline UNI 178 & $0.0 \%$ & $5.3 \%$ & $0.0 \%$ & $0.1 \%$ & $0.0 \%$ & $0.0 \%$ & $0.4 \%$ & $0.0 \%$ & $0.0 \%$ & $93.9 \%$ & $0.2 \%$ & $0.0 \%$ \\
\hline UNI 191 & $6.5 \%$ & $0.9 \%$ & $0.0 \%$ & $25.2 \%$ & $0.0 \%$ & $1.3 \%$ & $0.0 \%$ & $0.0 \%$ & $0.0 \%$ & $0.0 \%$ & $66.1 \%$ & $0.1 \%$ \\
\hline UNI 214 & $1.9 \%$ & $13.4 \%$ & $0.0 \%$ & $1.0 \%$ & $0.1 \%$ & $0.0 \%$ & $20.4 \%$ & $0.0 \%$ & $0.0 \%$ & $0.0 \%$ & $4.2 \%$ & $58.9 \%$ \\
\hline
\end{tabular}

789

790 Table 2. The similarity between three different datasets using Procrustes distance

791 (A) PC-DFA at the strain level

\begin{tabular}{|c|c|c|c|c|c|c|}
\hline $\begin{array}{l}\text { Averaging on ST } \\
\text { level }\end{array}$ & $\begin{array}{c}\text { FT-IR } \\
\text { (IS) }\end{array}$ & $\begin{array}{c}\text { FT-IR } \\
\text { (ST) }\end{array}$ & $\begin{array}{c}\text { Raman } \\
\text { (IS) }\end{array}$ & $\begin{array}{c}\text { Raman } \\
\text { (ST) }\end{array}$ & $\begin{array}{c}\text { MALDI } \\
\text { (IS) }\end{array}$ & $\begin{array}{c}\text { MALDI } \\
\text { (ST) }\end{array}$ \\
\hline FT-IR (IS) & - & & & & & \\
\hline FT-IR (ST) & 0.0858 & - & & & & \\
\hline Raman (IS) & 0.2125 & 0.2933 & - & & & \\
\hline Raman (ST) & 0.2314 & 0.3187 & 0.1502 & - & & \\
\hline MALDI (IS) & 0.8602 & 0.889 & 0.899 & 0.8202 & - & \\
\hline MALDI (ST) & 0.9125 & 0.8846 & 0.9149 & 0.8988 & 0.1812 & - \\
\hline
\end{tabular}

792

793 (B) PC-DFA at the isolate level

\begin{tabular}{|c|c|c|c|c|c|c|}
\hline $\begin{array}{l}\text { Averaging on IS } \\
\text { level }\end{array}$ & $\begin{array}{c}\text { FT-IR } \\
\text { (IS) }\end{array}$ & $\begin{array}{c}\text { FT-IR } \\
\text { (ST) }\end{array}$ & $\begin{array}{c}\text { Raman } \\
\text { (IS) }\end{array}$ & $\begin{array}{c}\text { Raman } \\
\text { (ST) }\end{array}$ & $\begin{array}{c}\text { MALDI } \\
\text { (IS) }\end{array}$ & $\begin{array}{c}\text { MALDI } \\
\text { (ST) }\end{array}$ \\
\hline FT-IR (IS) & - & & & & & \\
\hline FT-IR (ST) & 0.1085 & - & & & & \\
\hline Raman (IS) & 0.2112 & 0.2446 & - & & & \\
\hline Raman (ST) & 0.2411 & 0.3168 & 0.1132 & - & & \\
\hline MALDI (IS) & 0.8593 & 0.8719 & 0.8196 & 0.8001 & - & \\
\hline MALDI (ST) & 0.8975 & 0.8608 & 0.8841 & 0.8703 & 0.0681 & - \\
\hline
\end{tabular}

795 defined 12 types) and isolate (33 classes) levels, respectively. 